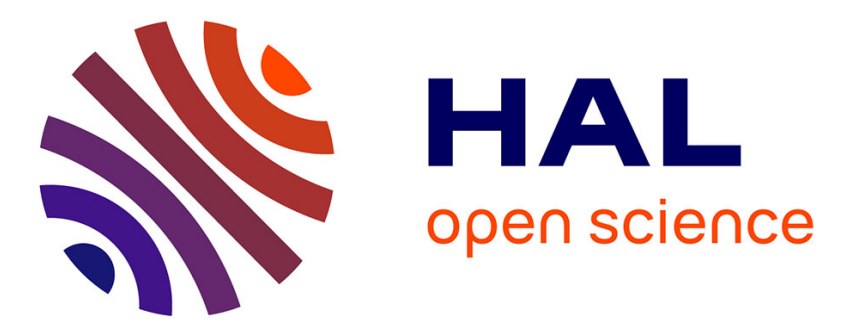

\title{
A conservative well-balanced hybrid SPH scheme for the shallow-water model
}

Christophe Berthon, Matthieu de Leffe, Victor Michel-Dansac

\section{To cite this version:}

Christophe Berthon, Matthieu de Leffe, Victor Michel-Dansac. A conservative well-balanced hybrid SPH scheme for the shallow-water model. FVCA VII, Jun 2014, Berlin, Germany. pp.817-825, 10.1007/978-3-319-05591-6_82. hal-00960982

\section{HAL Id: hal-00960982 \\ https://hal.science/hal-00960982}

Submitted on 19 Mar 2014

HAL is a multi-disciplinary open access archive for the deposit and dissemination of scientific research documents, whether they are published or not. The documents may come from teaching and research institutions in France or abroad, or from public or private research centers.
L'archive ouverte pluridisciplinaire HAL, est destinée au dépôt et à la diffusion de documents scientifiques de niveau recherche, publiés ou non, émanant des établissements d'enseignement et de recherche français ou étrangers, des laboratoires publics ou privés. 


\title{
A conservative well-balanced hybrid SPH scheme for the shallow-water model
}

\author{
Christophe Berthon, Matthieu de Leffe, Victor Michel-Dansac
}

\begin{abstract}
A scheme defined by a hybridization between SPH method and finite volume method is considered. The aim of the present communication is to derive a suitable discretization of the source term to enforce the required well-balanced property. To address such an issue, we adopt a relevant reformulation of the flux function by involving the free surface instead of the water height. Such an approach gives a natural discretization of the topography source term in order to preserve the lake at rest. Moreover, we prove that the scheme is in conservative form, which is, in general, a very difficult task since we do not impose restrictive assumptions on the SPH method. Several 1D numerical experiments are performed to exhibit the properties of the scheme.
\end{abstract}

\section{Introduction}

The present work concerns the numerical approximation of the well-known shallowwater model. The model under consideration is given as follows:

$$
\left\{\begin{array}{l}
\partial_{t} h+\partial_{x}(h u)=0 \\
\partial_{t}(h u)+\partial_{x}\left(h u^{2}+\frac{g}{2} h^{2}\right)=-h g \partial_{x} Z
\end{array}\right.
$$

\footnotetext{
Christophe Berthon

Laboratoire de Mathématiques Jean Leray, UMR 6629, 2 rue de la Houssinière, BP 92208, 44322

Nantes Cedex 3, France, e-mail: christophe.berthon@univ-nantes.fr

Matthieu de Leffe

HydrOcean, 1 rue de la Noë, CS 32122, 44321 Nantes Cedex 3, France, e-mail: matthieu.deleffe@hydrocean.fr

Victor Michel-Dansac

Laboratoire de Mathématiques Jean Leray, UMR 6629, 2 rue de la Houssinière, BP 92208, 44322

Nantes Cedex 3, France, e-mail: victor.michel-dansac@univ-nantes.fr
} 
where $h \geq 0$ denotes the water height, $u \in \mathbb{R}$ is the water velocity in the $x$ direction, and $g>0$ stands for the gravity constant. The function $Z$ denotes the smooth topography. To shorten the notations, the system is rewritten in the following form:

$$
\partial_{t} \Phi+\partial_{x} f(\Phi)=S, \quad \Phi=\left(\begin{array}{c}
h \\
h u
\end{array}\right), f(\Phi)=\left(\begin{array}{c}
h u \\
h u^{2}+\frac{g}{2} h^{2}
\end{array}\right), S=\left(\begin{array}{c}
0 \\
-h g \partial_{x} Z
\end{array}\right) .
$$

By adopting a finite volume method to approximate the weak solutions, a usual property to be satisfied concerns the lake at rest preservation. Indeed, the stationary solution given by $u=0$ and $h+Z=\mathrm{cst}$, must be exactly preserved by the numerical method (for instance, see $[2,5,3]$ and references therein).

Here, we do not consider a classic finite volume scheme, but we adopt a hybrid method deriving from the SPH techniques. More precisely, the SPH method (issuing from the particle methods) involves a like interface numerical flux function. According to [7, 14], this like interface flux function is substituted by a like finite volume flux function derived from approximate Riemann solvers [5, 12, 9].

In the present paper, we exhibit a source term discretization to make wellbalanced this hybrid numerical technique. The paper is organized as follows. In the next section, we briefly recall the gradient evaluation derived from the SPH technique, and the hybrid version by considering approximate Riemann solvers. Next, in Section 3, after [4], we adopt a relevant reformulation of the model to introduce a suitable well-balanced discretization of the topography source term. The full discrete scheme is proved to preserve the required lake at rest, and it is in conservation form without any additional assumptions. In Section 4, numerical experiments are performed in order to illustrate the relevance of the scheme. A short conclusion is given in the last section.

\section{Introduction to the SPH method and finite volume hybridization}

The Smoothed Particle Hydrodynamics (SPH) method was introduced to perform astrophysical simulations. Recent works (for instance see [13] and references therein) extend the SPH method in the field of CFD. Now, we present the derivation of the SPH scheme to approximate the weak solutions of (2).

First, it is worth noticing that, for all real functions $f: \mathbb{R} \rightarrow \mathbb{R}$, we have the following relation: $f(x)=(f * \delta)(x)=\int_{\mathbb{R}} f(y) \delta(x-y) d y$, with $\delta$ the Dirac measure. The particle approximation relies on a suitable regularization of this Dirac measure.

To address such as issue, after $[10,11]$, a kernel $W \in C_{0}^{1}(\mathbb{R}) \cap L^{1}(\mathbb{R})$ is introduced, which is usually some bell-shaped function, depending on both center $x$ and smoothing length $h$. It must satisfy the consistency conditions [11] given by $\int_{\mathbb{R}} W(x, h) d x=1$ and $\int_{\mathbb{R}} W^{\prime}(x, h) d x=0$.

Now, after $[10,11]$, the particle approximation of $f$, given by $(f * W)(x)=$ $\int_{\mathbb{R}} f(y) W(x-y, h) d y$, is nothing but a second-order accurate approximation of the 
function $f$. Using the Green formula, we easily deduce an approximation of $f^{\prime}$, given by $\int_{\mathbb{R}} f(y) W^{\prime}(x-y, h) d y$.

Unfortunately, this particle approximation involves integrals which cannot be exactly evaluated. As a consequence, a quadrature formula is adopted as follows: $\int_{\mathbb{R}} f(x) d x \simeq \sum_{j \in \mathscr{P}} \omega_{j} f_{j}$, where $x_{j}$ are the quadrature points, $f_{j}$ denotes the evaluation of $f$ at point $x_{j}$, and $\omega_{j}$ stands for the associated weight. Within the SPH method, the quadrature points are made of particles $x_{i}$ with volume $\omega_{i}$, and $\mathscr{P}$ denotes the set of interacting particles $x_{j}$ close enough to the particle $x_{i}$. We then get the following approximation:

$$
\Pi^{h}(f)_{i}=\sum_{j \in \mathscr{P}} \omega_{j} f_{j} W_{i j}, \quad \Pi^{h}\left(f^{\prime}\right)_{i}=\sum_{j \in \mathscr{P}} \omega_{j} f_{j} W_{i j}^{\prime}, \quad W_{i j}=W\left(x_{i}-x_{j}, h\right) .
$$

From now on, let us underline that a natural property to be satisfied by this particle approximation is $\Pi^{h}(1)=1$ and $\Pi^{h}\left(1^{\prime}\right)=0$, which reads

$$
\sum_{j \in \mathscr{P}} \omega_{j} W_{i j}=1 \quad \text { and } \quad \sum_{j \in \mathscr{P}} \omega_{j} W_{i j}^{\prime}=0 .
$$

Such relations are not always satisfied by usual choices for the kernel $W$ (see [13]).

By adopting the derivative discretization formula (3), the SPH scheme for a general set of equations (2) is given by

$$
\frac{1}{\Delta t} \omega_{i}\left(\Phi_{i}^{n+1}-\Phi_{i}^{n}\right)+\sum_{j \in \mathscr{P}} \omega_{i} \omega_{j}\left(f_{i}^{n}+f_{j}^{n}\right) W_{i j}^{\prime}=\omega_{i} S_{i}^{n},
$$

with $f_{i}^{n}=f\left(\Phi_{i}^{n}\right), \Delta t$ the time step, and $\omega_{i} \Phi_{i}^{n}$ the vector of conserved variables for the particle $x_{i}$.

Concerning the source term discretization $S_{i}^{n}$, one may adopt the particle approximation (3). However, in order to satisfy the required well-balanced property, we will introduce a specific approximation of the topography in the next section.

To conclude this brief presentation of the SPH scheme, we now show the finite volume approximate Riemann solver hybridization as introduced in [14]. Indeed, the derivative discrete operator involves an interface flux approximation given by $\frac{1}{2}\left(f_{i}^{n}+f_{j}^{n}\right)$. In [14], this flux approximation is substituted by the numerical flux function coming from usual Godunov-type scheme (for instance Godunov, HLL, HLLC, Roe scheme [8]). As a consequence, we consider the following modified hybrid SPH scheme:

$$
\left\{\begin{array}{l}
\frac{1}{\Delta t} \omega_{i}\left(h_{i}^{n+1}-h_{i}^{n}\right)+\sum_{j \in \mathscr{P}} 2 \omega_{i} \omega_{j}(h u)_{i j} W_{i j}^{\prime}=0 \\
\frac{1}{\Delta t} \omega_{i}\left(h_{i}^{n+1} u_{i}^{n+1}-h_{i}^{n} u_{i}^{n}\right)+\sum_{j \in \mathscr{P}} 2 \omega_{i} \omega_{j}\left(h u^{2}+\frac{g}{2} h^{2}\right)_{i j} W_{i j}^{\prime}=\omega_{i} S_{i},
\end{array}\right.
$$

where $f^{\Delta x}\left(\Phi_{i}^{n}, \Phi_{j}^{n}\right)=\left((h u)_{i j},\left(h u^{2}+\frac{g}{2} h^{2}\right)_{i j}\right)$ stands for the numerical flux function issuing from a usual finite volume scheme. 


\section{A well-balanced scheme}

Neither usual SPH techniques (for instance Monaghan SPH formulation [11], Vila formulation [13]) nor the here presented hybrid schemes combining SPH and Riemann solvers [14] are able to preserve the lake at rest steady state.

In order to derive a lake at rest preserving scheme, we adopt a recent equivalent reformulation of the PDE. After [7, 14], the flux function, which is in the center of the hybridization, is reformulated by considering the free surface $H=h+Z$ and the velocity. Indeed, within the required lake at rest, these two quantities stay constant, which is of prime importance in the numerical flux definition.

Here, we assume $H>0$ and we introduce $X=h / H$ a water height like fraction. To shorten the notations, we set $V={ }^{t}(H, H u)$. In the following statement, by considering $V$, we reformulate the system $(1)$ (see $[3,4])$.

Lemma 1. The weak solutions of (1) satisfy the following system:

$$
\left\{\begin{array}{l}
\partial_{t} h+\partial_{x}(X(H u))=0, \\
\partial_{t}(h u)+\partial_{x}\left(X\left(H u^{2}+\frac{g}{2} H^{2}\right)\right)=\frac{g}{2} \partial_{x}(h Z)-g h \partial_{x} Z
\end{array}\right.
$$

Let us emphasize that these reformulations involve the flux function but for the new variables $V$. As a consequence, as soon as a lake at rest is considered, this flux function only involves constant states (see (10) later on). This turns out to be the main ingredient to get the required well-balanced property.

Now, we suggest to adopt the hybrid scheme (5) but for the equivalent formulation (6). As a consequence, the hybrid SPH scheme under consideration now reads

$$
\left\{\begin{array}{l}
\frac{1}{\Delta t} \omega_{i}\left(h_{i}^{n+1}-h_{i}^{n}\right)+\sum_{j} 2 \omega_{i} \omega_{j} X_{i j}(H u)_{i j} W_{i j}^{\prime}=0 \\
\frac{1}{\Delta t} \omega_{i}\left(h_{i}^{n+1} u_{i}^{n+1}-h_{i}^{n} u_{i}^{n}\right)+\sum_{j} 2 \omega_{i} \omega_{j} X_{i j}\left(H u^{2}+g \frac{H^{2}}{2}\right)_{i j} W_{i j}^{\prime}= \\
\omega_{i}\left(\frac{g}{2} \partial_{x}(h Z)-g h \partial_{x} Z\right)_{i}
\end{array}\right.
$$

Concerning the here involved numerical flux function $\left((H u)_{i j},\left(H u^{2}+g H^{2} / 2\right)_{i j}\right)$, we directly adopt $f^{\Delta x}\left(V_{i}, V_{j}\right)$. To complete the scheme, we characterize the new formulation of the source term. Let us first notice the following easy relation:

$$
\frac{g}{2} \partial_{x}(h Z)-g h \partial_{x} Z=\frac{g}{2} \partial_{x}\left(H^{2} X(1-X)\right)-g H X \partial_{x}(H(1-X)) .
$$

In fact, a straightforward application of the SPH discretization is not relevant and we need to consider an additional correction term. We thus adopt the following SPH like discretization of the source term: 


$$
\begin{aligned}
\omega_{i}\left(\frac{g}{2} \partial_{x}(h Z)-g h \partial_{x} Z\right)_{i}= & \frac{g}{2} \sum_{j} 2 \omega_{i} \omega_{j}\left(X_{i j} H_{i j}-2 \bar{H}_{i} \bar{X}_{i}\right)\left(H_{i j}\left(1-X_{i j}\right)\right) W_{i j}^{\prime}+ \\
& g \sum_{j} 2 \omega_{i} \omega_{j} \bar{H}_{i}^{2} \bar{X}_{i}\left(1-\tilde{X}_{i}\right) W_{i j}^{\prime},
\end{aligned}
$$

where $\bar{H}_{i}, \bar{X}_{i}$ and $\tilde{X}_{i}$ are averages to be defined. Let us remark that the correction term $g \sum_{j} 2 \omega_{i} \omega_{j} \bar{H}_{i}^{2} \bar{X}_{i}\left(1-\tilde{X}_{i}\right) W_{i j}^{\prime}$ vanishes as soon as the kernel function satisfies the consistency conditions (4). This correction term is, in fact, a representation of zero.

Equipped with the hybrid SPH scheme (7)-(8), we now exhibit a suitable definition for $\bar{X}_{i}$ to enforce the expected well-balanced property.

Theorem 1. Assume both free surface averages to satisfy:

$$
H_{i j}=\bar{H}_{i}=H, \quad \text { as soon as } H_{i}=H_{j}=H .
$$

Assume $\bar{X}_{i}$ is defined by

$$
\bar{X}_{i}=\frac{1}{2} \frac{\sum_{j} \omega_{j} X_{i j}^{2} W_{i j}^{\prime}}{\sum_{j} \omega_{j}\left(X_{i j}-1\right) W_{i j}^{\prime}+\left(\tilde{X}_{i}-1\right) \sum_{j} \omega_{j} W_{i j}^{\prime}} .
$$

Then the scheme (7)-(8) preserves the lake at rest.

Proof. At time $t^{n}$, we assume the approximate solution $\Phi_{i}^{n}$ be given by the lake at rest. Then, for all $i$ in $\mathbb{Z}$, we have $h_{i}^{n}+Z_{i}=H$ a positive constant and $u_{i}^{n}=0$. The proof consists in establishing $\Phi_{i}^{n+1}=\Phi_{i}^{n}$. Since the numerical flux function $f^{\Delta x}$ is consistent, it preserves the constant states. Hence, we have the following sequence of equalities:

$$
f^{\Delta x}\left(\Phi_{i}^{n}, \Phi_{i+1}^{n}\right)=f^{\Delta x}\left(\left(\begin{array}{c}
H \\
0
\end{array}\right),\left(\begin{array}{c}
H \\
0
\end{array}\right)\right)=f\left(\begin{array}{c}
H \\
0
\end{array}\right)=\left(\begin{array}{c}
0 \\
g \frac{H^{2}}{2}
\end{array}\right) .
$$

From the water height evolution issuing from (7), we immediately get $h_{i}^{n+1}=h_{i}^{n}$. Next, concerning the discharge evolution, because of the consistency properties of the involved average functions, $H_{i j}$ and $\bar{H}_{i}$, the source term discretization (8) now reads

$$
\omega_{i}\left(\frac{g}{2} \partial_{x}(h Z)-g h \partial_{x} Z\right)_{i}=\frac{g}{2} H^{2} \sum_{j} 2 \omega_{i} \omega_{j}\left(\left(X_{i j}-2 \bar{X}_{i}\right)\left(1-X_{i j}\right)+\bar{X}_{i}\left(1-\tilde{X}_{i}\right)\right) W_{i j}^{\prime} .
$$

Finally, by definition of $\bar{X}_{i}$, given by (9), a straightforward computation gives $\omega_{i}\left(\frac{g}{2} \partial_{x}(h Z)-g h \partial_{x} Z\right)_{i}=\frac{g}{2} H^{2} \sum_{j} 2 \omega_{i} \omega_{j} X_{i j} W_{i j}^{\prime}$. As a consequence, the updated discharge, given by (7), gives $u_{i}^{n+1}=0$, and the proof is achieved.

Let us underline that the formula (9), to define $\bar{X}_{i}$, is consistent with an evaluation of $X$ at particle $x_{i}$. Indeed, from the SPH space derivative approximation (3), we notice that $\frac{1}{2} \sum_{j} \omega_{j} X_{i j}^{2} W_{i j}^{\prime}$ is consistent with $\frac{1}{2} \partial_{x} X^{2}$ while $\sum_{j} \omega_{j}\left(X_{i j}-1\right) W_{i j}^{\prime}$ is consistent with $\partial_{x}(X-1)$. Since $\sum_{j} \omega_{j} W_{i j}^{\prime}$ is consistent with zero, then $\bar{X}_{i}$ is consistent with $\frac{1}{2} \partial_{x} X^{2} / \partial_{x}(X-1)=X$. 
To conclude this section, we remark that the required well-balanced property is established independently of the definitions of $X_{i j}$ and $\bar{H}_{i}$. Here, we adopt the averages introduced in [3, 4]: $\bar{H}_{i}=h_{i}^{n}+Z_{i}$ and $X_{i j}=X_{i}$ if $(H u)_{i j}>0, X_{j}$ otherwise.

In fact, at this level, we notice that the proposed scheme satisfies an additional stronger property. Indeed, when adopting SPH type scheme to approximate the solution of homogeneous hyperbolic systems (i.e. with vanishing source term), in general it is not possible to preserve the constant solutions. By considering an initial data made of a uniform constant state, the SPH approach makes some particles move and the constant initial data is no longer preserved. Since the derived scheme is wellbalanced, it obviously preserves such constant solutions as soon as the topography is flat, i.e. $Z=$ cst. Moreover, we can exhibit a precise definition of the average functions to preserve the conservation form of the scheme: $\sum_{i \in \mathbb{Z}} \omega_{i} h_{i}^{n+1}=\sum_{i \in \mathbb{Z}} \omega_{i} h_{i}^{n}$ and $\sum_{i \in \mathbb{Z}} \omega_{i} h_{i}^{n+1} u_{i}^{n+1}=\sum_{i \in \mathbb{Z}} \omega_{i} h_{i}^{n} u_{i}^{n}$. The conservation of the water height is directly deduced from the evolution law for $h_{i}^{n+1}$ given by (7). Next, considering the updated formula for the discharge, we easily obtain

$$
\begin{aligned}
\sum_{i \in \mathbb{Z}} \omega_{i} h_{i}^{n+1} u_{i}^{n+1}= & \sum_{i \in \mathbb{Z}} \omega_{i} h_{i}^{n} u_{i}^{n}-g \sum_{i \in \mathbb{Z}} \omega_{i} \bar{H}_{i} \bar{X}_{i} \sum_{j} 2 \omega_{j} H_{i j}\left(1-X_{i j}\right) W_{i j}^{\prime}+ \\
& g \sum_{i \in \mathbb{Z}} \omega_{i} \bar{H}_{i}^{2} \bar{X}_{i}\left(1-\tilde{X}_{i}\right) \sum_{j} 2 \omega_{j} W_{i j}^{\prime}
\end{aligned}
$$

Now, we have to define the average functions $\left(H_{i j}, \bar{H}_{i}, \bar{X}_{i}\right.$ and $\left.\tilde{X}_{i}\right)$ such that the discharge conservation is recovered as soon as the topography function is a given constant $Z$. Of course, providing that the consistency conditions (4) holds true, we have just to consider average functions such that $H_{i j}\left(1-X_{i j}\right)=Z$.

If (4) is not satisfied, we enter the delicate problem of the inconsistency of the SPH technique. Let us assume that the average functions satisfy the following condition as soon as the topography is a given constant $Z$ : $H_{i j}\left(1-X_{i j}\right)=Z$ and $\bar{H}_{i}\left(1-\tilde{X}_{i}\right)=Z$. Then we immediately recover the expected conservation of the discharge. Such average functions can be easily obtained. For instance, let us set $\bar{H}_{i}=h_{i}^{n}+Z_{i}, \tilde{X}_{i}=h_{i}^{n} /\left(h_{i}^{n}+Z_{i}\right)$ and $H_{i j}=H_{i}$ if $(H u)_{i j}>0, H_{j}$ otherwise.

\section{Numerical experiments}

We now illustrate the relevance of the proposed SPH scheme. For all the tests, the computational domain is $[0,25], 200$ particles are used, and the gravity constant is equal to 9.81 .

To test the well-balanced property, we consider a topography defined by $Z(x)=$ $0.4 e^{(\sin (x)-1)}$. The initial conditions are $h(x, 0)+Z(x)=0.5$, and $u(x, 0)=0$.

Figure 1 shows that the free surface is unperturbed with an oscillating topography. The velocity is, as expected, close to 0 , up to $10^{-15}$. The perturbations appearing the in the velocity are of the order of magnitude of the machine precision, 

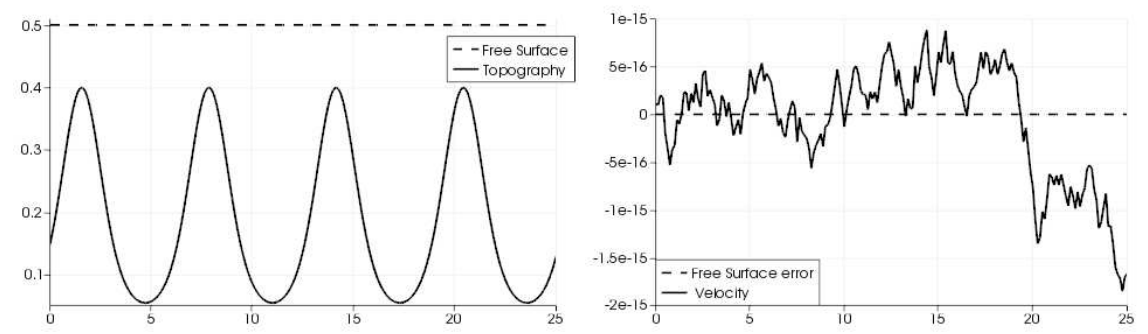

Fig. 1 Left: Free surface profile for the lake at rest with the above defined topography. Right: Velocity and free surface errors for this lake at rest. Both graphs show the solutions at time $t=600 \mathrm{~s}$.

which is confirmed by a simulation in quadruple precision, where the perturbations are close to 0 , up to $10^{-33}$.

The next three test cases come from [6]. The topography is flat with a bump for $x \in[8,12]$, as follows: $Z(x)=0.2-0.05(x-10)^{2}$. The transcritical flow without shock (G1), with shock (G2) and subcritical flow (G3) test cases are performed according to the initial and boundary conditions given by [6].
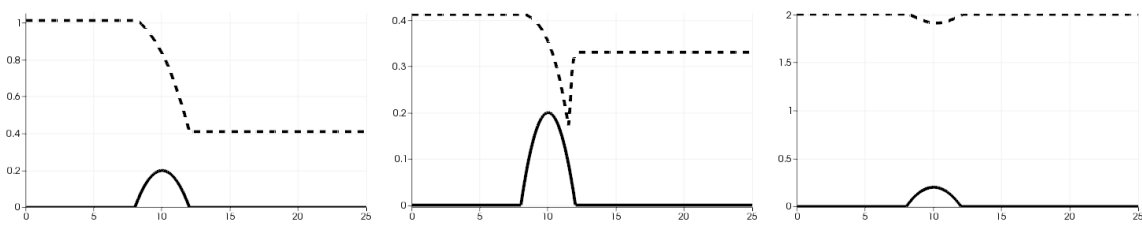

Fig. 2 From left to right, the three test cases defined in [6], respectively G1, G2, G3. The dashed line represents the free surface and the full line is the topography. The graphs show the solutions at time $t=600 s$.

Figure 2 shows good agreement with the exact results (see [6, 3] for instance).

In Table 1, the discharge errors turn out to be similar to other methods like hydrostatic reconstruction.

Table 1 Discharge errors for the three test cases described above. Comparisons between three schemes: the modified SPH scheme as well as the ones introduced in $[1,3]$.

\begin{tabular}{clccccc}
\hline Test case & \multicolumn{2}{l}{ Hydrostatic reconstruction } & \multicolumn{2}{c}{ Hydrostatic upwind } & \multicolumn{2}{c}{ SPH scheme } \\
\cline { 2 - 6 } & $L^{2}$ error & $L^{\infty}$ error & $L^{2}$ error & $L^{\infty}$ error & $L^{2}$ error $L^{\infty}$ error \\
\hline G1 & $4.35 \mathrm{E}-2$ & $1.92 \mathrm{E}-2$ & $5.98 \mathrm{E}-2$ & $1.87 \mathrm{E}-2$ & $5.67 \mathrm{E}-2$ & $1.85 \mathrm{E}-2$ \\
G2 & $4.88 \mathrm{E}-2$ & $3.31 \mathrm{E}-2$ & $4.68 \mathrm{E}-2$ & $2.85 \mathrm{E}-2$ & $5.50 \mathrm{E}-2$ & $4.02 \mathrm{E}-2$ \\
G3 & $9.62 \mathrm{E}-2$ & $3.07 \mathrm{E}-2$ & $9.78 \mathrm{E}-2$ & $2.70 \mathrm{E}-2$ & $9.83 \mathrm{E}-2$ & $2.74 \mathrm{E}-2$ \\
\hline
\end{tabular}




\section{Conclusion}

By adopting a suitable reformulation of the shallow-water model, we have derived a relevant discretization of the topography source term to enforce a hybrid SPH scheme (introduced in [7, 14]) to be well-balanced. Numerical simulations have been performed to illustrate the interest of such a topography source term discretization. Indeed, usual and hybrid SPH schemes are known to not preserve the constant state because of the kernel function which does not satisfy the consistency conditions (4). The proposed technique corrects such a failure.

Acknowledgements Victor Michel-Dansac would like to thank the project PEPS SPHINX of Labex AMIES (under contract ANR10-LABX-02). Christophe Berthon would like to thank the ANR-12-IS01-0004-01 GEONUM for financial support.

\section{References}

1. Audusse E., Bouchut F., Bristeau M. O., Klein R., Perthame B.: A fast and stable well-balanced scheme with hydrostatic reconstruction for shallow water ows SIAM J.Sci.Comp., 25, 20502065 (2004)

2. Bermudez A., Vazquez-Cendon M.E.: Upwind Methods for Hyperbolic Conservation Laws with Source Terms. Computers and Fluids. 23 1049-1071 (1994)

3. Berthon C., Foucher F.: Efficient well-balanced hydrostatic upwind schemes for shallow-water equations. J. Comput. Phys., 231 (15) (2012), pp. 4993-5015

4. Berthon C., Foucher F.: Hydrostatic upwind schemes for shallow-water equations. Finite Volumes for Complex Application VI, Springer Proceedings in Mathematics 4 (2011), pp. 97-106

5. Bouchut F.: Nonlinear stability of finite volume methods for hyperbolic conservation laws and well-balanced schemes for sources. Frontiers in Mathematics. Birkhuser Verlag, Basel, 2004.

6. Goutal N., Maurel F.: Proceedings of the 2nd workshop on dam-break wave simulation. Technical report, EDF-DER, HE-43/97/016/B (1997)

7. Guilcher P.M.: Contribution au développement d'une méthode SPH pour la simulation numérique des interactions houle-structure. Thèse de doctorat, École Centrale de Nantes, 2008

8. Harten A, Lax P., van Leer B.: On upstream differencing and Godunov-type schemes for hyperbolic conservation laws. SIAM Rev. 25 (1983), no. 1, 35-61.

9. Kröner D.: Finite volume schemes in multidimensions. Numerical analysis 1997 (Dundee), 179-192, Pitman Res. Notes Math. Ser., 380, Longman, Harlow, 1998.

10. Mas-Gallic S., Raviart P.A.: A Particle Method for First-order Symmetric Systems. Numerische Mathematik, 1987, Volume 51, Number 3, pp. 323-352

11. Monaghan J.J.: Smoothed Particles Hydrodynamics. Annual Review of Astronomy and Astrophysics, 1992, 30, pp. 543-574

12. Toro E.F.: Riemann solvers and numerical methods for fluid dynamics. Springer Verlag, Third Edition, A practical introduction, 2009

13. Vila J.P.: On particle weighted methods and smooth particle hydrodynamics. Math. Models Methods Appl. Sci., 09, 161 (1999)

14. Vila J.P.: SPH Renormalized Hybrid Methods for Conservation Laws: Applications to Free Surface Flows. Meshfree Methods for Partial Differential Equations II, (2005), pp. 207-229 